\title{
Caries risk assessment using Cariogram model among smokeless tobacco users in India
}

Nandini Sen ${ }^{1}$, Pratiksha Bathija ${ }^{2}$, Tulip Chakravarty ${ }^{1}$, Diptajit Das ${ }^{3}$,

1) Public Health Dentistry Department, Pacific Dental College and Hospital, Udaipur, India

2) Public Health Dentistry Department, Sri Aurobindo College of Dentistry \& P.G. Institute, Indore, India

3) Public Health Dentistry Department, Centre for Dental Education and Research, AIIMS, New Delhi, India

4) Oral and Maxillo-Facial Surgery Department, Jaipur Dental College, Jaipur, India

DOI: $10.15386 / \mathrm{mpr}-978$

Manuscript received: 05.02.2018

Received in revised form: 17.06.2018

Accepted: 12.11.2018

Address for correspondence: sen_ty31@yahoo.co.in

Nidhi Singh Baghel ${ }^{4}$, Tauseef Ahmad Khan ${ }^{4}$

\begin{abstract}
Background. Smokeless tobacco forms are known to have fermentable sugar compounds which may strengthen the development of cariogenic microbes. In addition, cervical abrasion of teeth occur at the site of tobacco pouch placement. These components may assume an essential role in caries advancement in smokeless tobacco users.
\end{abstract}

Objective. The objective of the study was to assess caries risk among smokeless tobacco users using Cariogram model.

Methods. A descriptive cross sectional study was conducted among 50 smokeless tobacco users of Udaipur for 3 months. Caries risk assessment was done by employing a proforma survey based on the Cariogram Model. Statistical analysis included descriptive statistics, Chi-square test and Stepwise multiple linear regression with $95 \%$ confidence interval and 5\% significance level.

Results. The majority of the smokeless tobacco users (46\%) were found to be in the "Moderate" Streptococcus mutans count category and portrayed "Moderate" plaque amount score (82\%). Smokeless tobacco users (34\%) depicted a higher caries risk profile than the control group (6\%) utilizing the Cariogram model.

Conclusion. Cariogram model could be a useful tool to represent caries risk among smokeless tobacco users.

Keywords: smokeless tobacco, dental caries, cariogram, caries risk, tobacco

\section{Introduction}

Globally tobacco usage enforces an enormous impact on public health and its burden is on the rise progressively. The World Health Organization predicts that deaths due to tobacco consumption may exceed 1.5 million yearly by 2020 [1]. In India, tobacco habit caused around 1 million deaths, i.e $10 \%$ of aggregate deaths in 2010, while in 2015, tobacco usage at 15-69 years age mounted from 79 million in 1998 to 108 million [2,3].

Smokeless tobacco (ST) utilization has unmistakable relationships with dental caries which can be attested to the fact that in the pouch and plug varieties of smokeless tobacco forms, sugar is usually found to be present [4-7]. Non-smoking forms of tobacco use have negative impacts on one's oral health producing caries susceptible conditions, which lead to dental caries with the passage of time.

A few studies confirmed an unknown relationship of tobacco use with dental caries [8-10]. A study by Mujahid et al (2014) on 96 patients of Andhra Pradesh, India, stated that the level of Streptococcus was appreciably higher among smokeless tobacco users [8]. Winn DM (2001) in his review article showed that caries incidence was higher in ST users [7]. Goin et al (1980) conducted an in-vivo study which showed that the count of microbes associated with root caries was higher on the root surface of teeth next to tobacco placement as compared to the teeth on the opposite side of the oral cavity [5].

In recent literature, caries risk assessment has acquired immense attention as a principal component in comprehensive management of the disease. In 1997, Dr. Bratthall introduced an interactive computer programme named Cariogram, which is the sole model capable of evaluating diverse factors like 
microflora, host response, oral health status and cariogenic diet involved in caries augmentation [11]. Thus, it is useful in assessing caries risk, as also shown in the studies by Raju et al (2016) and Celik et al (2012) [12,13].

But till now, no studies have been conducted to assess the caries risk among smokeless tobacco users using the Cariogram model. Hence, based on the scarce information available on the performance of Cariogram in adults and to fill the existing void in literature regarding the utility of Cariogram in determining caries risk in smokeless tobacco users, the present study was conducted with the aim to assess caries risk using Cariogram among smokeless tobacco users in India.

\section{Methods}

Study design, study area, duration and population

A descriptive cross sectional study was conducted among smokeless tobacco users visiting the outpatient department of a dental college and hospital, Udaipur city, Rajasthan, India, in the period May - July 2017. The Dental college was chosen as the study setting as it is situated in the peri-urban area and therefore subjects from both urban $\&$ rural areas could be incorporated.

\section{consent}

Ethical approval, official permission \& informed

The study protocol was reviewed and approved by the Institutional Review Board and the ethical committee of dental college and hospital and was granted ethical clearance. An informed consent was obtained for all the individuals who participated in the study before the start of the examination.

\section{Training and calibration}

Before the commencement of the study, the examiner was standardized and calibrated. The intra examiner reliability for Decayed Missing Filled Teeth (DMFT) index (1938) and Modified Plaque Index - Loe H. (1967) was assessed using Kappa statistics which was found to be $86 \%$ and $80 \%$ respectively.

\section{Inclusion criteria}

1. Age range $18-50$ years old.

2. Smokeless tobacco users- Subjects who had habit of chewing any form of tobacco more than 3 times a day for more than 1 year.

\section{Exclusion criteria}

Subjects with prosthesis, fixed orthodontic appliances, under medication which can alter salivary parameters, antibiotic therapy, history of radiotherapy, combination usage of tobacco, alcohol consumption, systemic disease, used mouthwash or undergone oral prophylaxis in past 1 month, uncooperative patients.

\section{Proforma details}

A survey proforma was designed which consisted of two sections:

Section I: General information - Demographic data including name, age, sex, adverse habits.

Section II: Information about Cariogram parameters

\section{Pilot survey}

A pilot study was conducted on 10 subjects, each of smokeless tobacco users and control group to determine the feasibility of the study, the time required for examination of each subject, to get acquainted to the laboratory procedure for salivary analysis and for sample size calculation.

\section{Sample size determination}

Based on the results obtained from the pilot study, sample size (n) was determined with 95\% confidence interval, 5\% allowable error and $80 \%$ power of the study using the following formula:

$$
\mathrm{n}=\left\{\mathrm{z}_{(1-\alpha) / 2}^{2} \mathrm{p}(1-\mathrm{p})\right\} / \mathrm{d}^{2}
$$

Final sample size was estimated to be 50 for Smokeless tobacco users and 50 subjects were included as Controls (normal healthy individuals without any history of habit).

\section{Methodology}

Study participants were selected by simple random sampling method based on inclusion and exclusion criteria. The investigator interviewed regarding socio-demographic data, diet frequency and fluoride exposure. Clinical examination was done to measure caries experience and plaque amount using DMFT (1938) and modified Plaque Index (Loe H.-1967) respectively.

Instruments used during examination, saliva flow rate and $\mathrm{pH}$ measurement were: plain mouth mirrors, explorers, tweezers, kidney trays, disposable mouth masks and gloves, cotton swabs, paraffin wax, plastic graduated test tubes, plastic funnels, measuring cups, Indikrom $\mathrm{pH}$ indicating papers.

\section{Salivary parameters estimation}

Salivary flow rate estimation was done by collecting stimulated saliva sample within the morning hours (between 10.00 AM - 11.30 AM) to maintain circadian rhythm. The study participants were asked to spit in a funnel connected to a graduated test tube after every 1 minute for 5 minutes and expressed in $\mathrm{ml} / \mathrm{min}$. Colorimetric method using Indikrom papers was used to estimate the buffer capacity of saliva.

\section{Microbiological analysis}

Using an inoculation loop, sample was streaked on Mitis salivarius bacitracin agar (MSB) medium, which is selective for mutans streptococci and on Rogosa SL agar medium for lactobacilli. The colony counts were made using electron microscope and expressed in number of 
CFU (Colony Forming Units) per ml of saliva.

\section{Assessment of caries risk profile}

Since India is a developing nation and vulnerable for dental caries, 'High risk' category was opted for Country. For every individual, the Cariogram parameters were given a score and entered into the Cariogram computer program to obtain the individual caries risk profile. The software produces a pie diagram which represents the percentages of 'Diet' (Dark Blue), 'Bacteria' (Red), 'Susceptibility' (Light Blue), 'Circumstances' (Yellow). These 4 sectors dictate the percentage of 'Chance to avoid new caries' (Green) sector. Hence, the bigger the green sector, the lesser is the risk of caries and vice versa.

\section{Statistical analysis}

The recorded data and Cariogram software data were compiled and entered in Microsoft Excel 2007 and then exported to data editor page of SPSS version 20.0 (SPSS Inc., Chicago, Illinois, USA). Descriptive statistics included computation of percentages and Chi-Square Test $(\chi 2)$ was applied. Stepwise multiple linear regression analysis was done to estimate the relationship between Caries risk as dependent variable and other factors as independent variables. For all tests, confidence interval and p-value were set at $95 \%$ and $\leq 0.05$ respectively.

\section{Results}

Demographic data showed that smokeless tobacco users group comprised of 47 males (94\%) and 3 females (6\%) (Table I).

Table II depicts the comparative assessment of study participants based on parameters used in Cariogram model. The majority of the smokeless tobacco users $[\mathrm{n}=29(58 \%)]$ showed "low" caries experience. The DMFT scores were statistically significantly associated with the study groups $(\mathrm{p}=0.001)$.

The study results depicted that most of the smokeless tobacco users $[\mathrm{n}=41(82 \%)]$ portrayed "Moderate" plaque amount score whereas among the controls it was found that $36(72 \%)$ study participants were in "Low" plaque amount category.

The maximum number of smokeless tobacco users $[n=23(46 \%)]$ were mainly found to be in the "Moderate" S. mutans count category while most of the controls depicted a "Very Low" count $[\mathrm{n}=44(88 \%)]$. The majority of the subjects in both the groups used fluoridated toothpaste regularly ( $62 \%$ and $68 \%$ respectively).

Both the study groups depicted normal saliva secretion ( $66 \%$ and $94 \%$ respectively). It was observed that majority of the smokeless tobacco users had "Adequate" $(\geq 6.0)$ salivary $\mathrm{pH}(56 \%)$.

The best predictors in the descending order for caries risk were buffer capacity followed by DMFT score, saliva flow rate with the variances $8.1 \%, 10.9 \%$ and $12.1 \%$ respectively (Table III).

Smokeless tobacco users $[\mathrm{n}=17(34 \%)]$ showed higher caries risk profile than controls $[n=3(6 \%)]$ group (Figure 1).

Figure 2 represents the mean percentage of individual sector in Cariogram contributing to caries risk among the smokeless tobacco users. Susceptibility sector was highest in the contribution of caries risk followed by bacteria, circumstances and diet sectors.

Table I. Distribution of study population according to gender.

\begin{tabular}{l|c|c|c} 
& $\begin{array}{c}\text { Smokeless Tobacco Users } \\
\text { n (\%) }\end{array}$ & $\begin{array}{c}\text { Control Group } \\
\text { n (\%) }\end{array}$ & $\begin{array}{c}\text { Total } \\
\text { n (\%) }\end{array}$ \\
\hline Male & $47(94)$ & $45(90)$ & $92(92)$ \\
Female & $3(6)$ & $5(10)$ & $8(8)$ \\
Total n (\%) & $\mathbf{5 0}(\mathbf{1 0 0})$ & $\mathbf{5 0 ( 1 0 0 )}$ & $\mathbf{1 0 0 ( 1 0 0 )}$
\end{tabular}


Table II. The comparative assessment of study participants based on parameters used in Cariogram model.

\begin{tabular}{|c|c|c|c|}
\hline & $\begin{array}{c}\text { Smokeless Tobacco Users } \\
\text { n ( }(\%)\end{array}$ & $\begin{array}{c}\text { Control Group } \\
\text { n (\%) }\end{array}$ & p-value \\
\hline \multicolumn{4}{|l|}{ Caries experience Score } \\
\hline Low (DMFT 1) & $29(58.0)$ & $11(22.0)$ & \multirow{2}{*}{$0.001 *$} \\
\hline Moderate (DMFT 2) & $8(16.0)$ & $5(10.0)$ & \\
\hline Very low ( $\leq 10^{3} \mathrm{CFU} / \mathrm{ml}$, very low sugar consumption) & $36(72.0)$ & $44(88.0)$ & \multirow{4}{*}{$0.001 *$} \\
\hline Low ( $10^{4} \mathrm{CFU} / \mathrm{ml}$, low sugar consumption) & $13(26.0)$ & $6(12.0)$ & \\
\hline Moderate ( $10^{5} \mathrm{CFU} / \mathrm{ml}$, moderate sugar consumption) & $1(2.0)$ & 0 & \\
\hline High ( $\geq 10^{6} \mathrm{CFU} / \mathrm{ml}$, high sugar consumption) & 0 & 0 & \\
\hline Maximum 7 & $1(2.0)$ & $1(2.0)$ & \multirow{2}{*}{$0.001 *$} \\
\hline$>7$ & 0 & 0 & \\
\hline \multicolumn{4}{|l|}{ Plaque Amount Score } \\
\hline Very low (0-Excellent) & $1(2.0)$ & $11(22.0)$ & \multirow{4}{*}{$0.001 *$} \\
\hline Low (0.1-0.9 Good) & $6(12.0)$ & $36(72.0)$ & \\
\hline Moderate (1.0-1.9 Fair) & $41(82.0)$ & $3(6.0)$ & \\
\hline High (2.0-3.0 Poor) & $2(4.0)$ & 0 & \\
\hline \multicolumn{4}{|l|}{ Mutans Streptococci Score } \\
\hline Very low ( $\leq 20,000 \mathrm{CFU} / \mathrm{ml}$ Saliva $)$ & $8(16.0)$ & $44(88.0)$ & $0.001 *$ \\
\hline Additional Fluoride measures & 0 & $2(4.0)$ & \multirow{3}{*}{0.262} \\
\hline Fluoride toothpaste only & $31(62.0)$ & $34(68.0)$ & \\
\hline No fluoride & $19(38.0)$ & $14(28.0)$ & \\
\hline \multicolumn{4}{|l|}{ Saliva Secretion Amount } \\
\hline Normal & $33(66.0)$ & $47(94.0)$ & \multirow{4}{*}{$0.001 *$} \\
\hline Low (0.9-1.1 ml Stimulated) & $17(34.0)$ & $3(6.0)$ & \\
\hline Low (0.5-0.9 ml Stimulated) & 0 & 0 & \\
\hline Very Low ( $\leq 0.5 \mathrm{ml}$ Saliva $)$ & 0 & 0 & \\
\hline \multicolumn{4}{|l|}{ Saliva Buffer capacity } \\
\hline Adequate $(\mathrm{pH} \geq 6.0)$ & $28(56.0)$ & $44(88.0)$ & \multirow{4}{*}{$0.001 *$} \\
\hline Reduced (pH 4.5-5.5) & $22(44.0)$ & $5(10.0)$ & \\
\hline Low $(\mathrm{pH} \leq 4.0)$ & 0 & $1(2.0)$ & \\
\hline Total & $50(100)$ & $50(100)$ & \\
\hline
\end{tabular}

Test applied: Chi-square test; *p $\leq 0.05$ (statistically significant)

Table III. Stepwise multiple linear regression analysis with caries risk as the dependent variable.

\begin{tabular}{|c|c|c|c|c|}
\hline Model & $\mathbf{R}$ & $\mathbf{R 2}$ & F - value & p - value \\
\hline \multicolumn{5}{|c|}{ Caries Risk } \\
\hline 1 & 0.301 (a) & 0.081 & 9.782 & 0.002 (a) \\
\hline 2 & $0.357(b)$ & 0.109 & 0.037 & $0.046(b)$ \\
\hline 3 & $0.387(\mathrm{c})$ & 0.121 & 0.012 & $0.047(\mathrm{c})$ \\
\hline \multicolumn{5}{|c|}{ a. Predictors: (Constant), Buffer capacity } \\
\hline \multicolumn{5}{|c|}{ b. Predictors: (Constant), Buffer capacity, DMFT score } \\
\hline \multicolumn{5}{|c|}{ c. Predictors: (Constant), Buffer capacity, DMFT score, Saliva flow rate } \\
\hline
\end{tabular}




\section{Caries Risk}

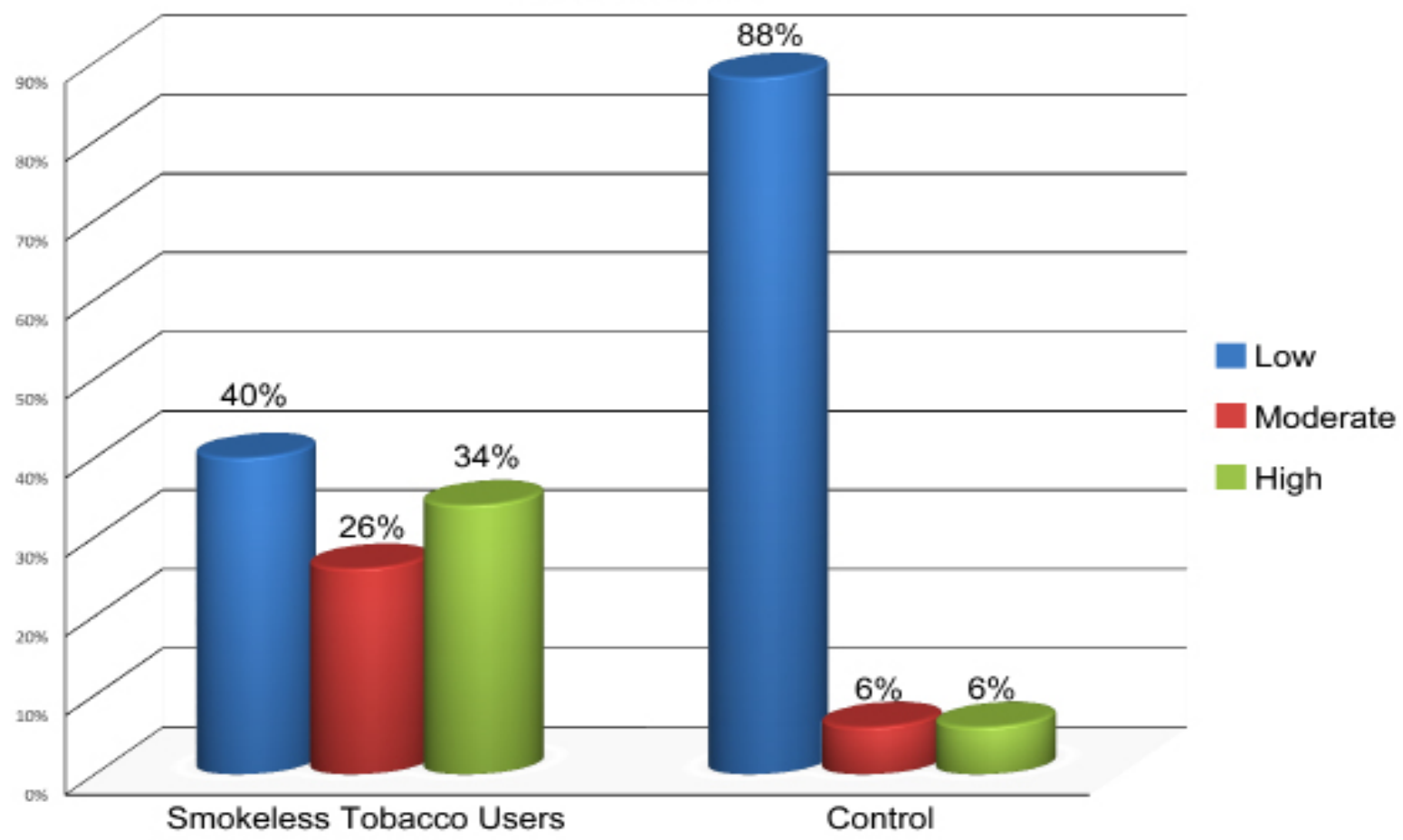

Figure 1. Comparative assessment of the study population based on caries risk

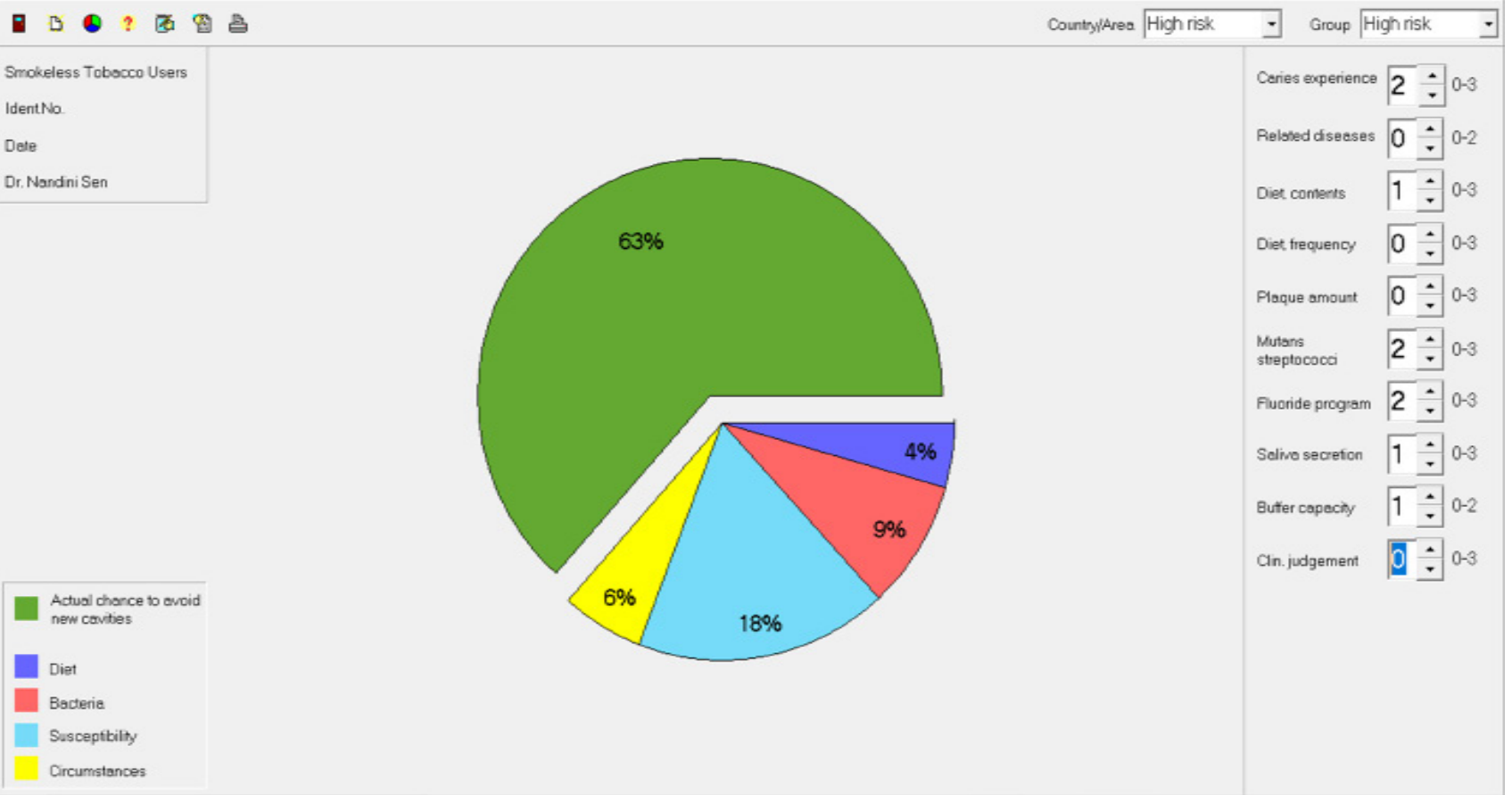

Figure 2. Mean percentage of individual sector in Cariogram contributing to caries risk among smokeless tobacco users group. 


\section{Discussion}

Mankind has witnessed the detrimental effects of tobacco consumption on general and oral health for decades. Apart from being a carcinogen, it is known to cause different ailments including being a risk factor for dental caries [14]. To the best of our knowledge, our study is the first one of its kind to have utilized the innovative Cariogram model in the identification and comparison of caries risk among smokeless tobacco users.

In the current study, the majority were males in the smokeless tobacco users group, which is in agreement with the studies conducted by Rooban et al (2011) and Mohamed and Janakiram (2013) $[15,16]$. But conversely, a study by Lashkari and Shukla (2016) in Karnataka, India showed that females constituted half of the study population (45.9\%) [17]. Among the majority of Indian population due to cultural barriers, women have a natural tendency to hide, even if they were engaged in certain adverse habits like tobacco chewing, which explains our demographic results with almost no females.

Our study results revealed that smokeless tobacco users (58\%) had higher mean DMFT scores than controls, which is in accordance with the findings by Lashkari and Shukla (2016) and Hans et al (2014) [17,18]. Nagarajappa et al (2010) showed in their research that the use of chewing tobacco decreased the Lactobacilli colony-forming units count. Similarly in the present study, the results showed that more than half of the smokeless tobacco users $(72 \%)$ had very low lactobacilli count [19]. A plausible reason might be that India is a developing country and most of the people belong to low or middle socioeconomic strata which restricts their usage of refined confectionary sugar products.

Streptococcus mutans count was significantly associated with the smokeless tobacco consumers $(\mathrm{p}=0.001)$ with majority $[\mathrm{n}=23(46 \%)]$ showing 'Moderate' count in the current study which is similar to the study by Mujahid et al (2014), where the mean Streptococcal count was maximum in the tobacco chewers with dental caries group [8].

We observed in the present study that the majority (65\%) of the participants of the study groups used fluoridated toothpaste as a routine, while less than half of the study population (33\%) had no fluoride usage. Less usage of fluoride in our study population may have led to higher caries risk which can be attributed to the lack of awareness and preventive programmes conducted at community level.

Tobacco usage in various forms have been found to affect the salivary parameters, consequently leading to oral diseases. The present study results portrayed that Smokeless tobacco users had lower saliva flow rate $[\mathrm{n}=17$ $(34 \%)]$ and $\mathrm{pH}[\mathrm{n}=22(44 \%)]$ than the controls which is congruent with a handful of studies conducted by Grover et al. (2016), Chakrabarty et al. (2016) and Gopal et al. (2016) [20-22].
In the present study, the low salivary flow rate and reduced $\mathrm{pH}$ amongst the smokeless tobacco users could be attested to the higher DMFT scores amongst them. Possible reason for the above fact might be that the flushing action of saliva is impaired as the salivary flow rate decreases, which leads to accumulation of food debris and also acidic $\mathrm{pH}$ favors microbial growth which consequently may have contributed to higher caries prevalence among the smokeless tobacco users.

In the current study, results showed that the mean percentage of the various Cariogram sectors (Diet, Bacteria, Susceptibility, Circumstances) contributing to dental caries were higher for the smokeless tobacco users than the control group. In particular, the Susceptibility sector was the chief contributor for smokeless tobacco users which is indicative of infrequent fluoride exposure, reduced saliva flow and $\mathrm{pH}$ attributing to increased caries risk.

Overall findings of the present study are in accordance with certain former studies conducted by Winn and Tomar (1999), Lashkari and Shukla (2016) and Holmen et al. (2013) where the authors confirmed that Smokeless tobacco users were at higher caries risk than control group since high levels of added fermentable sugar and sweetening compounds are present in non-smoking tobacco forms which are capable of stimulating the growth of cariogenic bacteria and also cervical abrasion of teeth could occur at the site of tobacco pouch placement increasing the chances of root caries $[5,14,17,23,24]$. This addition is presumed to have a neutralizing effect on the bitter taste of tobacco [25]. Stepwise multiple linear regression analysis depicted that Buffer capacity was the strongest predictor of caries risk.

However, the study exhibits certain limitations that needs to be addressed. Since the Cariogram model was used, a preset number of caries risk factors as mentioned in the Cariogram manual were recorded. Other factors such as the effect of tobacco (nicotine), proper diet history, oral hygiene practices were not considered. The study results are based on self-report, therefore tobacco consumption related responses may not be accurate. The current study considered caries risk evaluation among the smokeless tobacco users only, therefore studies comparing the caries risk of smokers and smokeless tobacco users should be conducted.

\section{Conclusion}

Tobacco use predisposes an individual to diverse oral ailments, including dental caries and hence, caries risk assessment has gained utmost importance in recent times. The study confirmed that Cariogram model could be a useful tool to represent caries risk among Smokeless tobacco users. The findings from the different cariogram elements in this study converged to indicate that Smokeless tobacco users were at a higher caries risk as compared to the control group. It is recommended that tobacco cessation and awareness programmes concerning the deleterious 
effects of tobacco should be conducted mandatorily to reduce its consumption. Moreover we recommend to carry out similar studies separately among rural and urban population to perceive the impact of factors prevailing in these areas which can affect dental caries prevalence.

\section{Acknowledgements}

The authors would like to thank the study participants for their participation and kind cooperation throughout the study.

\section{References}

1. Rani M, Bonu S, Jha P, Nguyen SN, Jamjoum L. Tobacco use in India: prevalence and predictors of smoking and chewing in a national cross sectional household survey. Tob Control. 2003; 12:e4.

2. Jha P, Jacob B, Gajalakshmi V, Gupta PC, Dhingra N, Kumar $\mathrm{R}$, et al. A nationally representative case-control study of smoking and death in India. N Engl J Med. 2008;358:1137-1147.

3. Mishra S, Joseph RA, Gupta PC, Pezzack B, Ram F, Sinha $\mathrm{DN}$, et al. Trends in bidi and cigarette smoking in India from 1998 to 2015, by age, gender and education. BMJ Global Health. 2016; 1:e000005.

4. Weintraub JA, Burt BA. Periodontal effects and dental caries associated with smokeless tobacco use. Public Health Rep. 1987; 102:30-35.

5. Going RE, Hsu SC, Pollack RL, Haugh LD. Sugar and fluoride content of various forms of tobacco. J Am Dent Assoc. 1980;100:27-33.

6. Offenbacher S, Weathers DR. Effects of smokeless tobacco on the periodontal, mucosal and caries status of adolescent males. J Oral Pathol. 1985;14:169-181.

7. Winn DM. Tobacco use and oral disease. J Dent Educ. 2001;65:306-312.

8. Mujahid M, Anushree B, Shobha M. Salivary levels of cariogenic streptococcus and lactobacillus among tobacco abusers in Andhra Pradesh, India. Research Journal of Pharmaceutical, Biological and Chemical Sciences. 2014;5:521-530.

9. Singh M, Ingle NA, Kaur N, Yadav P, Ingle E. Effect of longterm smoking on salivary flow rate and salivary $\mathrm{pH}$. J Indian Assoc Public Health Dent. 2015;13:11-13.

10. Voelkar MA, Simmer-Beck M, Cole M, Keeven E, Tira D. Preliminary findings on the correlation of saliva $\mathrm{pH}$, buffering capacity, flow, Consistency and Streptococcus mutans in relation to cigarette smoking. J Dent Hyg. 2013;87:30-37.
11. Bratthall D, Hänsel Petersson G. Cariogram--a multifactorial risk assessment model for a multifactorial disease. Community Dent Oral Epidemiol. 2005;33:256-264.

12. Raju SH, Fareed N, Sudhir KM, Krishna Kumar R. Caries risk assessment among subjects with periodontal disease using cariogram study model. OHDM 2016;15:223-228.

13. Celik EU, Gokay N, Ates M. Efficiency of caries risk assessment in young adults using Cariogram. Eur J Dent 2012;6:270-279.

14. Vellappally S, Fiala Z, Smejkalová J, Jacob V, Shriharsha P. Influence of tobacco use in dental caries development. Cent Eur J Public Health. 2007;15:116-121.

15. Rooban T, Vidya K, Joshua E, Rao A, Ranganathan S, Rao UK, et al. Tooth decay in alcohol and tobacco abusers. J Oral Maxillofac Pathol. 2011;15:14-21.

16. Mohamed S, Janakiram C. Periodontal status among tobacco users in Karnataka, India. Indian J Public Health. 2013;57:105108.

17. Lashkari KP, Shukla A. Prevalence of dental caries among smokeless tobacco chewers in Dakshina Kannada district population: A Cross Sectional Study. OHDM. 2016;15:1-3.

18. Hans R, Thomas S, Dagli RJ, Solanki J, Arora G. Prevalence of dental caries among prisoners of Central Jail, Jodhpur City, Rajasthan, India. World Journal of Dentistry. 2014;5:92-97.

19. Nagarajappa S, Prasad KV. Oral microbiota, dental caries and periodontal status in smokeless tobacco chewers in Karnataka, India: a case-control study. Oral Health Prev Dent. 2010;8:211219.

20. Grover N, Sharma J, Sengupta S, Singh S, Singh N, Kaur H. Long-term effect of tobacco on unstimulated salivary $\mathrm{pH}$. J Oral Maxillofac Pathol. 2016;20:16-19.

21. Chakrabarty S, Patil S, Bandalore SR, Kempegowda RT, Shivu, Pewa S. A comparative study of long-term effect of tobacco on resting whole mouth salivary flow rate and $\mathrm{pH}$. J Indian Acad Oral Med Radiol. 2015;27:549-552.

22. Saraswathi Gopal K, Amala M, Harshavardhan BG. Effect of smoking and chewing tobacco on resting salivary flow rate and $\mathrm{ph}$. International Journal of Current Research 2016;8:44344-44348.

23. Tomar SL, Winn DM. Chewing tobacco use and dental caries among U.S. men. J Am Dent Assoc. 1999;130:1601-1610.

24. Holmén A, Strömberg U, Magnusson K, Twetman S. Tobacco use and caries risk among adolescents--a longitudinal study in Sweden. BMC Oral Health. 2013;13:31.

25. Talhout R, Opperhuizen A, van Amsterdam JG. Sugars as tobacco ingredient: effects on mainstream smoke composition. Food Chem Toxicol. 2006;44:1789-1798. 